\section{Identification of a Class of Nonlinear Deterministic Systems with Application to Manipulators}

Shir-Kuan Lin

Abstract- This note presents a recursive identification method to estimate the minimal parameters of a class of nonlinear deterministic system. The result can be applied to a manipulator with unknown torque constants. A procedure to identify the torque constants, the friction, and the gravity parameters at the same time is then proposed. This is worthwhile since the PD feedback control with the gravity and friction compensation ensures a zero steady-state response for the set-point control. A theory addresses the minimal parameters composed of the three groups of parameters. The identification procedure for the minimal parameters is conducted by moving one joint with a constant velocity at a time. The persistently exciting trajectories for the identification are also suggested. The experiment on the PUMA 560 illustrates the identification method.

\section{INTRODUCTION}

This note deals with the parameter identification of the following deterministic system

$$
\boldsymbol{i}=\operatorname{diag}\left(\eta_{1}, \cdots, \eta_{n}\right) \boldsymbol{A}(\boldsymbol{q}) \boldsymbol{w}
$$

where $\operatorname{diag}\left(\eta_{i}, \cdots, \eta_{n}\right)$ is a diagonal matrix with the diagonal entries of $\eta_{i}, \cdots, \eta_{n}$ and $\boldsymbol{A ( q )}$, as shown by (2), found at the bottom of the next page, $\boldsymbol{q} \in R^{m}$, and subscripts $L(i)$ and $U(i)$ are the integer variables to label the columns of $\boldsymbol{A}$. Note that $L(1)=1$ and $L(i+1)=U(i)+1$. Suppose that the number of columns of $\boldsymbol{A}$ is $p$, i.e., $p=U(n)$. The entries of $\boldsymbol{A}$ (i.e., $a_{i j}, i=1, \cdots, n$, $j=1, \cdots, p)$ are all functions of $q$. In system $(1), i$ is the input vector, $\boldsymbol{q}$ is the vector of state variables, and $\eta_{i}$ and $w_{j}$ (elements of $\boldsymbol{w})$ are constant parameters. Assume that $a_{i, L(i)}(\boldsymbol{q}), \cdots, a_{i, U(i)}(\boldsymbol{q})$ and one of $a_{i j}(\boldsymbol{q}), j>U(i)$, are linearly independent over $R^{m}$ and the elements of $\boldsymbol{w}$ are independent parameters. If all $\eta_{i}$ are known a priori, the parameter identification of system (1) is a simple least squares problem since the columns of $\boldsymbol{A}$ are linearly independent. The elements of $\boldsymbol{w}$ form a set of minimal parameters for determining the system dynamics since they are identifiable and are linearly independent [17] and [18]. The problem will not be so easy, however, when $\eta_{i}$ are unknown.

We often encounter this problem in mechanical systems such as robotic manipulators. It has been shown [2] that the actuator forces of a manipulator are linear with respect to the inertia parameters and in the form of $\tau=\boldsymbol{A}(\boldsymbol{q}) \boldsymbol{w}$, where $\tau, \boldsymbol{q}$ and $\boldsymbol{w}$ are, respectively, the actuator forces, the joint displacements, and the inertia parameters. Many works [3], [8], [10], [13], [18], [20], [24]-[28], and [32] proposed various methods to identify the minimal combinations of the manipulator inertia parameters. Most of manipulator control systems, however, send the current commands to the servo amplifiers, which are linearly proportional to the actuator forces (or torques). In this sense, the manipulator dynamic should be modeled by (1) with $1 / \eta_{i}$ as the torque constants. The motor torque constants provided by the manufacturer are usually of low precision, their values will even vary with the use time [28].

Manuscript received October 30, 1992; revised July 1993 and September 10,1993 . This work was supported by the National Science Council, Taiwan, under Grant NSC82-0422-E-009-070.

The author is with the Institute of Control Engineering, National Chiao Tung University, Hsinchu 30050, Taiwan.

IEEE Log Number 9402735.

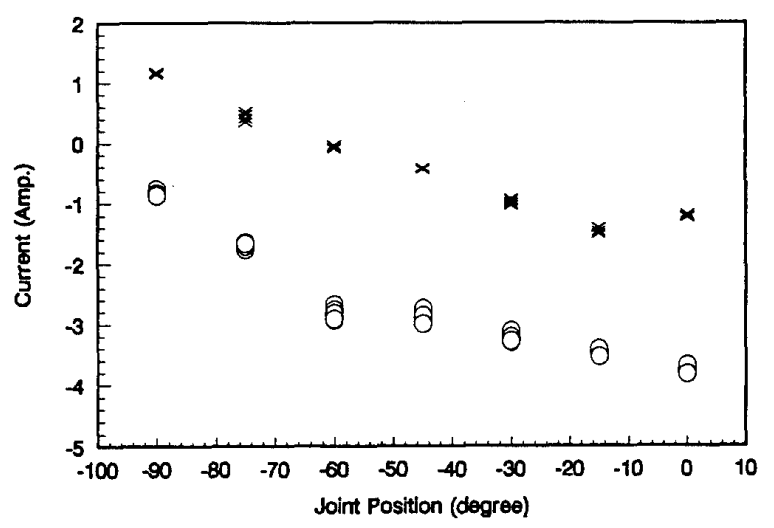

Fig. 1. Repeated experiments for the minimal current required to start a single joint (joint 2 of PUMA 560) in motion.

There are two approaches to this problem in the robotic literature. Atkeson et al. [2] used a six-axis force-torque sensor on a lever arm to statically measure the force at the end of the lever arm acted by the manipulator and then to calibrate the torque constants. Raucent et al. [27], [28], and West et al. [31] avoided the torque constant problem by measuring the reaction forces and moments on a sensing platform on which the manipulator stands. Although both require extra equipment, the latter does not provides the torque constants that are necessary in the implementation of the computed torque control scheme.

In this note, we first solve the parameter identification problem of system (1) and then apply the result to the manipulator. It is found that (1) can be converted to a recursive form which turns out to be a standard least squares problem again. Although some works [7], [9], [10], [17], [18], [21]-[23] have tried to formulate $\boldsymbol{A}$ and/or $\boldsymbol{w}$ of a manipulator for parameter identification, the results are very complicated. For the sake of simplicity, only the inertia parameters required for the gravity load are considered in the context. There is no doubt that the minimal parameters for the gravity load can be extracted from those [7], [9], [18], [21], [22] and [23] for the full dynamics; however, no one really exploited the result for the gravity case. Furthermore, we find that including the friction does not destroy the original theory. The gravity parameters (i.e., the inertia parameters for gravity load), torque constants, and the friction coefficients, in combined forms, can then be identified simultaneously. The identification process is to move only one joint with a constant velocity at a time.

Mayeda et al. [20] and Yoshida et al. [32] also proposed a static test to estimate the gravity parameters. The torque command of a joint is gradually changed until the joint starts to move. The average of the torque commands for both directions of a joint is taken as the gravity load, the bias is seen as friction. After knowing the gravity parameters, they then estimated the symmetric Coulomb and viscous friction. The static test has two drawbacks: tedious operation procedure and large error bounds. Fig. 1 shows the repeated measurement data for the static test on the joint 2 of the PUMA 560 $\mathrm{arm}$. The repeatability of the static friction is not well. This is one of the reasons why we propose an alternative method to identify these parameters at the same time. Besides, we adopt the experience of Armstrong [1] and Canudas et al. [4] to model the friction with a nonsymmetric Coulomb friction and viscous friction. 
Wen and Bayard [30] have rigorously shown that a PD feedback controller with the gravity compensation can asymptotically stabilize the manipulator with zero steady-state error for set-point commands. The identified values of this note are suitable to the set-point control (i.e., regulation), which will be verified by an experiment. Since most practical tasks of an industrial robot are composed of the set-point commands, the present identification method is very valuable.

This note is organized as follows. Section II discusses the identification theory for the deterministic system (1). The application of the theory to a manipulator with unknown torque constants is addressed in Section III. The identification experiment on the PUMA 560 arm is presented in Section IV.

Definition: A set of columns $\boldsymbol{a}_{i}(\boldsymbol{\theta}): R^{m} \rightarrow R^{n}$ is said to be linearly dependent over $R^{m}$ if there exist constants $\alpha_{i}, i=1, \cdots, n$, not all zero such that $\sum_{i=1}^{n} \alpha_{i} \boldsymbol{a}_{i}(\boldsymbol{\theta})=\mathbf{0}, \forall \boldsymbol{\theta} \in R^{m}$. If $\alpha_{i}$ are all zero, the set is said to be linearly independent over $R^{m}$.

\section{MaIN REsUlt}

System (1) can be reformed to

$$
i=A^{*} w^{*}
$$

where the entries of $\boldsymbol{A}^{*}$ and the elements of $\boldsymbol{w}^{*}$ are, respectively

$$
\begin{gathered}
a_{i j}^{*}= \begin{cases}0 & \text { for } j<L(i) \\
a_{i j} & \text { for } L(i) \leq j \leq U(i) \\
\frac{\eta_{i}}{\eta_{k}} a_{i j} & \text { for } L(k) \leq j \leq U(k), k>i\end{cases} \\
w_{j}^{*}=w_{j} \eta_{k} \text { for } L(k) \leq j \leq U(k), k=1, \cdots, n .
\end{gathered}
$$

The nonlinear deterministic system (3) can be decoupled to the recursive linear deterministic forms of

$$
\begin{aligned}
& i_{n}=\left[\begin{array}{lll}
a_{n, L(n)} & \cdots & a_{n, U(n)}
\end{array}\right]\left[\begin{array}{c}
w_{L(n)}^{*} \\
\vdots \\
w_{U(n)}^{*}
\end{array}\right] \\
& i_{i}=\left[\begin{array}{llllll}
a_{i, L(i)} & \cdots & a_{i, U(i)} & b_{i, i+1} & \cdots & b_{i, n}
\end{array}\right]\left[\begin{array}{c}
w_{L(i)}^{*} \\
\vdots \\
w_{U(i)}^{*} \\
\eta_{i} / \eta_{i+1} \\
\eta_{i} / \eta_{i+2} \\
\vdots \\
\eta_{i} / \eta_{n}
\end{array}\right] \\
& =\left[\begin{array}{llll}
a_{i, L(i)} & \cdots & a_{i, U(i)} & \nu_{i}
\end{array}\right]\left[\begin{array}{c}
w_{L(i)}^{*} \\
\vdots \\
w_{U(i)}^{*} \\
\eta_{i} / \eta_{i+1}
\end{array}\right]
\end{aligned}
$$

where $b_{i j}=\sum_{k=L(j)}^{U(j)} a_{i k} w_{k}^{*}$ for $j>i$ and $\nu_{i}=b_{i, i+1}+$ $\sum_{j=i+2}^{n} b_{i j}\left(\eta_{i+1} / \eta_{j}\right)$. It can be shown that if one of $a_{i j}, L(i+1) \leq$ $j \leq U(n)$, is linearly independent of $a_{i, L(i)}, \cdots, a_{i, U(i)}$, so is $\nu_{i}$ by the definition. Equations (6) and (7) then imply the following theorem.
Theorem 1: For the class of nonlinear deterministic system (1), if $a_{i, L(i)}(\boldsymbol{q}), \cdots, a_{i, U(i)}(\boldsymbol{q})$ and, at least, one of $a_{i j}(\boldsymbol{q}), j>U(i)$ are linearly independent over $R^{m}$, and the elements of $w$ are independent parameters, the elements of $\boldsymbol{w}^{*}$ and $\eta_{1} / \eta_{2}, \cdots, \eta_{n-1} / \eta_{n}$ form a set of minimal parameters for the system.

Proof: Because these parameters are independent and can be identified recursively from $n$ to one by using (6), (7), and the least squares method, the claim is true.

Q.E.D.

If $\nu_{i+1}=\nu_{i+2}=\cdots=\nu_{k-1}=0$ for $k-1>i$ (i.e., $a_{j, L(j+1)}=\cdots=a_{j, U(n)}=0$ for $\left.j=i+1, \cdots, k-1\right)$ then $\eta_{i+1} / \eta_{i+2}, \eta_{i+1} / \eta_{i+3}, \cdots, \eta_{i+1} / \eta_{k}$ cannot be identified by the above recursive procedure, so that $\nu_{i}$ cannot be calculated out. This renders (7) useless. Alternatively (7) can be reformed as

$i_{i}=\left[\begin{array}{llllllll}a_{i, L(i)} & \cdots & a_{i, U(i)} & b_{i, i+1} & b_{i, i+2} & \cdots & b_{i, k-1} & \nu_{i}^{*}\end{array}\right]$

$$
\left[\begin{array}{c}
w_{L(i)}^{*} \\
\vdots \\
w_{U(i)}^{*} \\
\eta_{i} / \eta_{i+1} \\
\eta_{i} / \eta_{i+2} \\
\vdots \\
\eta_{i} / \eta_{k-1} \\
\eta_{i} / \eta_{k}
\end{array}\right]
$$

where $\nu_{i}^{*}=b_{i, k}+\sum_{j=k+1}^{n} b_{i j} \eta_{k} / \eta_{j}$. Therefore, we can still get the same result that is stated as follows.

Corollary 2: For the same system described in Theorem 1, if $\nu_{i+1}=\nu_{i+2}=\cdots=\nu_{k-1}=0$ (but $\nu_{i} \neq 0, \nu_{k} \neq 0$ ), and additionally there are some elements among $a_{i, L(j)}, \cdots, a_{i, U(j)}$ for every $j, i<j<k$, that are linearly independent of, at least, one element of every other group of $a_{i, L(m)}, \cdots, a_{i, U(m)}, i<m<k$ and $m \neq j$, then the minimal parameters in Theorem 1 are still the minimal parameters.

If $\nu_{1}=\nu_{2}=\cdots=\nu_{k-1}=0$, but $\nu_{k} \neq 0$, then $\eta_{1} / \eta_{2}, \eta_{2} / \eta_{3}, \cdots, \eta_{k-1} / \eta_{k}$ are redundant parameters.

\section{APPLICATION to Manipulators}

In this section, we illustrate the application of the above theory to a manipulator. For the sake of simplicity, only the gravity load is taken into account in the modeling of the manipulator, i.e., the Coriolis and centrifugal dynamics are ignored.

\section{A. Dynamic Model}

We consider a manipulator with $n$ low-pair joints, which are labeled as joint 1 to $n$ outward from the base. Assign a body-fixed frame on each joint (i.e., frame $E_{i}$ is fixed on joint $i$ ) in accord with the normal driving-axis coordinate system [16] (known also as modified Denavit-Hartenberg notation [6]). The distance from the origin of $E_{i}$ to that of $E_{j}$ is designated as ${ }_{i}^{j} s$ and that to the center of mass of link $i$ as $\boldsymbol{c}_{i}$.

In the normal driving-axis coordinate system (see Fig. 2), the zaxis of a body-fixed frame is the driving axis of the corresponding link, i.e., the unit vector along joint $i$ is $\boldsymbol{u}_{i}^{\langle i\rangle}=[0,0,1]^{T}$, where superscript " $\langle i\rangle$ " denotes the representation of a vector with respect

$$
\boldsymbol{A}(\boldsymbol{q}) \equiv\left[\begin{array}{ccccccccccc}
a_{1, L(1)} & \cdots & a_{1, U(1)} & a_{1, L(2)} & \cdots & a_{1, U(2)} & \cdots & \cdots & a_{1, L(n)} & \cdots & a_{1, U(n)} \\
0 & \cdots & 0 & a_{2, L(2)} & \cdots & a_{2, U(2)} & \cdots & \cdots & a_{2, L(n)} & \cdots & a_{2, U(n)} \\
\vdots & \vdots & \vdots & \vdots & \vdots & \vdots & \vdots & \vdots & \vdots & \vdots & \vdots \\
0 & \cdots & \cdots & \cdots & \cdots & \cdots & \cdots & 0 & a_{n, L(n)} & \cdots & a_{n, U(n)}
\end{array}\right]
$$




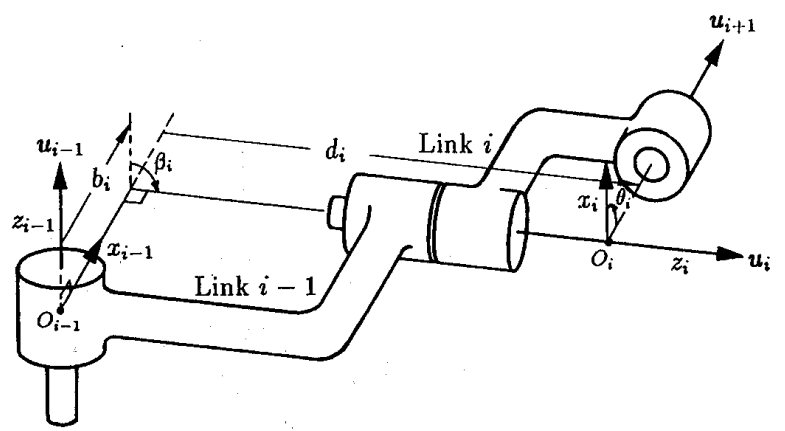

Fig. 2. Normal driving-axis coordinate system.

to frame $E_{i}$. The distance from the origin of frame $E_{i-1}$ to frame $E_{i}$ is shown to be

$$
\begin{aligned}
{ }_{i-1}^{i} s^{\langle i-1\rangle}=\left[\begin{array}{c}
b_{i} \\
-d_{i} \sin \beta_{i} \\
d_{i} \cos \beta_{i}
\end{array}\right] \text { or } \\
{ }_{i-1}^{i} s^{\langle i\rangle}=\left[\begin{array}{c}
b_{i} \cos \theta_{i} \\
-b_{i} \sin \theta_{i} \\
d_{i}
\end{array}\right]
\end{aligned}
$$

where $b_{i}, d_{i}, \beta_{i}$ and $\theta_{i}$ are the geometrical parameters of the coordinate system and shown in Fig. 2. The coordinate transformation matrix from $E_{i-1}$ to $E_{i}$ is then

$$
{ }_{i-1}^{i} \boldsymbol{R}=\left[\begin{array}{ccc}
\cos \theta_{i} & -\sin \theta_{i} & 0 \\
\cos \beta_{i} \sin \theta_{i} & \cos \beta_{i} \cos \theta_{i} & -\sin \beta_{i} \\
\sin \beta_{i} \sin \theta_{i} & \sin \beta_{i} \cos \theta_{i} & \cos \beta_{i}
\end{array}\right] .
$$

The composite body $i$ is defined as the union of link $i$ to link $n$. Let the mass of the composite body $i$ and the first moment of the composite body about the origin of $E_{i}$ be denoted as $\hat{m}_{i}$ and $\hat{c}_{i}$, respectively (i.e., $\hat{m}_{i}=\sum_{j=i}^{n} m_{j}$ and $\hat{c}_{i}^{\langle i\rangle}=\sum_{j=i}^{n} m_{j}\left({ }_{i}^{j} \boldsymbol{s}^{\langle i\rangle}+\boldsymbol{c}_{j}^{\langle i\rangle}\right)$, where $m_{j}$ is the mass of link $j$ ).

When the manipulator remains stationary, the actuator forces sustain the gravity only, and are then named stationary actuator forces. Applying statics, we get the required stationary actuator force and then the armature current of joint $i$ as

$$
\frac{i_{i}}{\eta_{i}}=\frac{C_{i} i_{i}}{r_{i}}=K_{i}^{*} \hat{\boldsymbol{c}}_{i}^{(i\rangle} \cdot\left[\begin{array}{c}
-\left(\boldsymbol{g}^{(i)}\right)_{y} \\
\left(\boldsymbol{g}^{(i)}\right)_{x} \\
0
\end{array}\right]-K_{i} \hat{m}_{i}\left(\boldsymbol{g}^{\langle i\rangle}\right)_{z}
$$

where $(\cdot)_{x}$ denotes the $\mathrm{x}$-component of a representation, $r_{i}$ is the gear ratio, $i_{i}$ and $C_{i}$ are, respectively, the armature current and the torque constant of the actuator motor driving joint $i, \eta_{i}=r_{i} / C_{i}$ is the ratio of the gear ratio to the torque constant, $g$ is the gravitational acceleration, and

$$
K_{i}^{*} \equiv 1-K_{i} \equiv \begin{cases}1, & \text { for rotational joint } i \\ 0, & \text { for translational joint } i\end{cases}
$$

In the present problem, the kinematic parameters are known $a$ priori. There are four unknown gravity parameters $m_{i}$ and $m_{i} c_{i}^{\langle i\rangle}$ for each link. Not all of them are identifiable, however, since the actuator forces are determined only by some combinations of these parameters. It is shown by the principle of induction [17] and [18] that

$$
\hat{c}_{i}^{\langle i\rangle}=\boldsymbol{k}_{i}+\boldsymbol{l}_{i}
$$

where

$$
\begin{aligned}
\boldsymbol{k}_{i}=m_{i} \boldsymbol{c}_{i}^{\langle i\rangle}+ & K_{i+1}^{*}\left(\hat{m}_{i+1}{ }_{i}^{i+1} \boldsymbol{s}^{\langle i\rangle}+{ }_{i}^{i+1} \boldsymbol{R}\left[\begin{array}{c}
0 \\
0 \\
\left(\boldsymbol{k}_{i+1}\right)_{z}
\end{array}\right]\right) \\
& +K_{i+1}\left(\hat{m}_{i+1}\left[\begin{array}{c}
\left({ }^{i+1} \boldsymbol{s}^{\langle i\rangle}\right)_{x} \\
0 \\
0
\end{array}\right]+{ }^{i+1} \boldsymbol{R} \boldsymbol{k}_{i+1}\right)
\end{aligned}
$$

$$
\begin{aligned}
\boldsymbol{l}_{i}=\boldsymbol{K}_{i+1}^{* *}{ }_{i}^{i+1} \boldsymbol{R}\left[\begin{array}{c}
\left(\hat{\boldsymbol{c}}_{i+1}^{\langle i+1\rangle}\right)_{x} \\
\left(\hat{\boldsymbol{c}}_{i+1}^{\langle i+1\rangle}\right)_{y} \\
\left(\boldsymbol{l}_{i+1}\right)_{z}
\end{array}\right] \\
+\boldsymbol{K}_{i+1}{ }^{i+1} \boldsymbol{R}\left(\boldsymbol{l}_{i+1}+\hat{m}_{i+1}\left[\begin{array}{c}
0 \\
0 \\
\left({ }^{i+1} \boldsymbol{s}_{i}^{\langle i+1\rangle}\right)=
\end{array}\right]\right) .
\end{aligned}
$$

Note that $\boldsymbol{k}_{n}=m_{n} \boldsymbol{c}_{n}^{\langle n\rangle}, \boldsymbol{l}_{n}=\mathbf{0}$, and $\left({ }_{i}^{i+1} \boldsymbol{s}^{\langle i+1\rangle}\right)_{z}=d_{i+1}$.

All constant terms of $\hat{\boldsymbol{c}}_{i}^{\langle i\rangle}$ are concatenated to be $\boldsymbol{k}_{i}$, while $\boldsymbol{l}_{i}$ collects the variant terms. As the x- and y-components of $K_{j}^{-*} \boldsymbol{k}_{j}$ and $K_{j} \hat{m}_{j}, u=i+1, \cdots, n$ are given, $\boldsymbol{l}_{i}$ can be calculated using the recursive form (14) and (15) from link $n$ to link 1 for any stationary configuration of the manipulator. For the detailed description of $\boldsymbol{k}_{i}$ and $l_{i}$, the reader is referred to [17] and [18].

Let joint $r$ be the first rotational joint counted from the base, joint $s$ be the nearest rotational joint not parallel to joint $r$. It was shown in [17] and [19] that when the manipulator stays stationary, the armature currents of the actuator motors can be described in a form of

$$
\operatorname{diag}\left(1 / \eta_{1}, \cdots, 1 / \eta_{n}\right) \boldsymbol{i}=\boldsymbol{A w}
$$

where $\boldsymbol{A}$ is defined in (2), and $\boldsymbol{w}$ consists of all nonzero elements in the same order as

$$
\boldsymbol{w}=\left[\begin{array}{c}
\delta_{1} K_{1}^{*}\left(\boldsymbol{k}_{1}\right)_{x} \\
\delta_{1} K_{1}^{*}\left(\boldsymbol{k}_{1}\right)_{y} \\
\sigma_{1} K_{1} \hat{m}_{1} \\
\vdots \\
\delta_{n} K_{n}^{*}\left(\boldsymbol{k}_{n}\right)_{x} \\
\delta_{n} \boldsymbol{K}_{n}^{*}\left(\boldsymbol{k}_{n}\right)_{y} \\
\sigma_{n} K_{n} \hat{m}_{n}
\end{array}\right] .
$$

Note that $\delta_{i}$ and $\sigma_{i}$ are either one or zero to denote the redundancy of the gravity parameters, which are defined as follows: $\delta_{i}=0$ for $r \leq i<s$ and $\boldsymbol{u}_{r} / / \boldsymbol{g}$, otherwise $\delta_{i}=1$. On the other hand, $\sigma_{i}=0$ for $\boldsymbol{u}_{i} \perp \boldsymbol{g}, \forall \boldsymbol{q} \in R^{n}$ (if $r<i<s$ for translational joint $i, \boldsymbol{u}_{i}$ is always perpendicular to $\boldsymbol{g}$ only when $\boldsymbol{u}_{r} / / \boldsymbol{g}$ or when $\boldsymbol{u}_{r} \perp \boldsymbol{g}$ and $\boldsymbol{u}_{i} / / \boldsymbol{u}_{r}$; while this can happen for $i>s$ only if $\boldsymbol{u}_{r} / / \boldsymbol{g}, \boldsymbol{u}_{s} \perp \boldsymbol{u}_{r}$ and $\boldsymbol{u}_{i} / / \boldsymbol{u}_{j} / / \boldsymbol{u}_{s}$ for any rotational joint $\left.j, s \leq j<i\right)$, otherwise $\sigma_{i}=1$.

It should be remarked that the elements (other than the ones associated with $K_{i} \hat{m}_{i}$ [see (16) and (17)]) of the rows of $\boldsymbol{A}$ corresponding to $i_{i} / \eta_{i}$ for all translational joints $i$ are all zero [19]. Applying Theorem 1 and Corollary 2 to (16), we get the following.

Theorem 3: When the manipulator stays stationary, a set of the minimal parameters for determining the armature currents $i$ for $\delta_{r} \neq 0$ is $\left\{\delta_{i} \boldsymbol{K}_{i}^{*} \eta_{i}\left(\boldsymbol{k}_{i}\right)_{x}, \delta_{i} K_{i}^{*} \eta_{i}\left(\boldsymbol{k}_{i}\right)_{y}, \sigma_{i} K_{i} \eta_{i} \hat{m}_{i}, \eta_{j} / \eta_{j+1}, i=\right.$ $1, \cdots, n, j=r, \cdots, n-1\}-\{0\}$. If $\delta_{r}=0$, then $\eta_{r} / \eta_{r+1}, \cdots, \eta_{s-1} / \eta_{s}$ are redundant parameters. 


\section{B. Friction}

The above derivation is based on the assumption that the friction is negligible or can be extracted from the manipulator dynamics To perform an identification on a manipulator with high gear ratios, the friction should be taken into account. It is of evidence in the robotics literature [1] and [15] that the friction in a manipulator can be approximated by the Coulomb friction and the viscous friction. The former is constant while the latter is proportional to the joint shaft velocity. The strategy for the identification experiment is to implement a stiff PID controller [1] to get the manipulator motion with a constant joint velocity. Specially, our identification procedure for the minimal parameters described in Theorem 3 will be conducted by only one joint moving (rotating or translating) at a time. Under such circumstances, the inertia effect and the coupling Coriolis and centrifugal effects do not appear in the dynamics. Therefore, the dynamical equations (11) for the stationary manipulator are still valid for the present case by adding a friction term, i.e.,

$$
\frac{i_{i}}{\eta_{i}}=K_{i}^{-*} \hat{\boldsymbol{c}}_{i}^{\langle i\rangle} \cdot\left[\begin{array}{c}
-\left(\boldsymbol{g}^{\langle i\rangle}\right)_{y} \\
\left(\boldsymbol{g}^{(i)}\right)_{x} \\
0
\end{array}\right]-K_{i} \hat{m}_{i}\left(\boldsymbol{g}^{\langle i\rangle}\right)_{z}+\frac{\tau_{f i}}{r_{i}}
$$

where $\tau_{f i}$ is the friction force of joint $i$. Note that the friction forces at a joint are internal forces and will not be propagated to the other joints. According to Theorem 3, (18) leads to

$$
\left[\begin{array}{c}
i_{1} \\
i_{2} \\
\vdots \\
i_{n}
\end{array}\right]=\operatorname{diag}\left(\eta_{1}, \cdots, \eta_{n}\right) A w+\left[\begin{array}{c}
\tau_{f 1} / C_{1} \\
\tau_{f 2} / C_{2} \\
\vdots \\
\tau_{f n} / C_{n}
\end{array}\right] .
$$

It is evident that the minimal parameters in Theorem 3 are still the minimal parameters of the system (19) if $\tau_{f i} / C_{i}, i=1, \cdots, n$, are known a priori. In fact, the friction terms and the minimal parameters in Theorem 3 can be identified at the same time. In this note, we adopted the nonsymmetric friction model [1] and [4]

$$
\frac{\tau_{f i}}{C_{i}}= \begin{cases}\lambda_{1 i}^{+}+\lambda_{2 i}^{+} \dot{q}_{i} & \text { for } \dot{q}_{i}>0 \\ \lambda_{1 i}^{-}+\lambda_{2 i}^{-} \dot{q}_{i} & \text { for } \dot{q}_{i}<0\end{cases}
$$

where $\lambda_{1 i}^{+}, \lambda_{2 i}^{+}, \lambda_{1 i}^{-}$, and $\lambda_{1 i}^{-}$are constants.

\section{Identification Algorithm}

Theorem 3 motivates us to introduce the new notations

$$
\begin{gathered}
\hat{m}_{i}^{*} \equiv \eta_{i} \hat{m}_{i} \\
\boldsymbol{k}_{i}^{*} \equiv \eta_{i} \boldsymbol{k}_{i} \\
\boldsymbol{l}_{i}^{*} \equiv \eta_{i+1} \boldsymbol{l}_{i} .
\end{gathered}
$$

Equation (15) leads to

$$
\begin{aligned}
& \boldsymbol{l}_{i}^{*}=\boldsymbol{K}_{i+1}^{*}{ }_{i}^{i+1} \boldsymbol{R}\left(\left[\begin{array}{c}
\left(\boldsymbol{k}_{i+1}^{*}\right)_{x} \\
\left(\boldsymbol{k}_{i+1}^{*}\right)_{y} \\
0
\end{array}\right]+\frac{\eta_{i+1}}{\eta_{i+2}} \boldsymbol{l}_{i+1}^{*}\right) \\
& +\boldsymbol{K}_{i+1}{ }^{i+1} \boldsymbol{R}\left(\hat{m}_{i+1}^{*}\left[\begin{array}{c}
0 \\
0 \\
\left({ }^{i+1}{ }_{i} \boldsymbol{s}^{\langle i+1\rangle}\right)_{z}
\end{array}\right]+\frac{\eta_{i+1}}{\eta_{i+2}} \boldsymbol{l}_{i+1}^{*}\right) .
\end{aligned}
$$

Note that $\boldsymbol{l}_{n}^{*}=\mathbf{0}$. The next step is to get the recursive linear deterministic forms like (7) for the manipulator system (19). Substituting (13) and (21)-(23) into (18) yields

$$
\begin{aligned}
i_{i}= & K_{i}^{*}\left(\boldsymbol{k}_{i}^{*}+\frac{\eta_{i}}{\eta_{i+1}} \boldsymbol{l}_{i}^{*}\right) \cdot\left[\begin{array}{c}
-\left(\underline{\boldsymbol{g}}^{\langle i\rangle}\right)_{y} \\
\left(\boldsymbol{g}^{\langle i\rangle}\right)_{x} \\
0
\end{array}\right] \\
& -K_{i} \hat{m}_{i}^{*}\left(\boldsymbol{g}^{\langle i\rangle}\right)_{z}+\tau_{f i} / C_{i} \\
= & K_{i}^{*}\left[\begin{array}{lll}
-\left(\boldsymbol{g}^{\langle i\rangle}\right)_{y} & \left(\boldsymbol{g}^{\langle i\rangle}\right)_{x} & \nu_{i}
\end{array}\right]\left[\begin{array}{c}
\left(\boldsymbol{k}_{i}^{*}\right)_{x} \\
\left(\boldsymbol{k}_{i}^{*}\right)_{y} \\
\eta_{i} / \eta_{i+1}
\end{array}\right] \\
& -K_{i}\left(\boldsymbol{g}^{\langle i\rangle}\right)_{z} \hat{m}_{i}^{*}+\tau_{f i} / C_{i}
\end{aligned}
$$

where

$$
\begin{gathered}
\nu_{i}=\left\{\begin{array}{c}
{\left[\begin{array}{cc}
-\left(\boldsymbol{g}^{\langle i\rangle}\right)_{y} & \left(\boldsymbol{g}^{\langle i\rangle}\right)_{x}
\end{array}\right]\left[\begin{array}{c}
\left(\boldsymbol{l}_{i}^{*}\right)_{x} \\
\left(\boldsymbol{l}_{i}^{*}\right)_{y}
\end{array}\right]} \\
\text { for } K_{i+1}^{*}=1 \\
b_{i, i+1}+b_{i, i+2} \frac{\eta_{i+1}}{\eta_{i+2}}+\cdots+b_{i, k-1} \frac{\eta_{i+1}}{\eta_{k-1}}+\nu_{i}^{*} \frac{\eta_{i+1}}{\eta_{k}} \\
\text { for } K_{i+1}=\cdots=K_{k-1}=K_{k}^{*}=1
\end{array}\right. \\
b_{i j}=\left[\begin{array}{lll}
-\left(\boldsymbol{g}^{\langle i\rangle}\right)_{y} & \left(\boldsymbol{g}^{\langle i\rangle}\right)_{x} & 0
\end{array}\right]_{i}^{j} \boldsymbol{R}\left[\begin{array}{c}
0 \\
0 \\
\hat{m}_{j}^{*}\left({ }_{j-1}^{j} \boldsymbol{s}^{\langle j\rangle}\right)_{z}
\end{array}\right] \text { for } j>i
\end{gathered}
$$

Equation (25) is equivalent to either (7) or (8). However, $\nu_{i}$ or $\nu_{i}^{*}$ can be calculated recursively without knowing $a_{i j}$.

For convenience, we introduce superscript "(i)" to denote the data measured from the $i$ th sampling point. Suppose that joint $k$ is the next rotational joint behind joint $i$. Let $\boldsymbol{w}_{i}^{*} \equiv$ $\left[\delta_{i}\left(\boldsymbol{k}_{i}^{*}\right)_{x}, \delta_{i}\left(\boldsymbol{k}_{i}^{*}\right)_{y}, \delta_{i}\left(\eta_{i} / \eta_{i+1}\right), \cdots, \delta_{i}\left(\eta_{i} / \eta_{k}\right), \lambda_{1 i}^{+}, \lambda_{2 i}^{+}, \lambda_{1 i}^{-}, \lambda_{2 i}^{-}\right]^{T}$ and $\boldsymbol{w}_{i} \equiv\left[\sigma_{i} \hat{m}_{i}^{*}, \lambda_{1 i}^{+}, \lambda_{2 i}^{+}, \lambda_{1 i}^{-}, \lambda_{2 i}^{-}\right]^{T}$. In an identification process with data of $m$ sampling points, (25) is then written, according to Theorem 3, as

$$
\left[\begin{array}{c}
i \mathbb{1}_{i} \\
\vdots \\
i \dot{\oplus}_{i}
\end{array}\right]=K_{i}^{*} \boldsymbol{P}_{i}^{*} \boldsymbol{w}_{i}^{*}+K_{i} \boldsymbol{P}_{i} \boldsymbol{w}_{i}
$$

where the $\ell$ th rows of $\boldsymbol{P}_{i}^{*}$ and $\boldsymbol{P}_{i}$ (denoted by $\boldsymbol{p}_{i ; l}^{*}$ and $\boldsymbol{p}_{i ; l}$, respectively) are (30) (at the bottom of the page) and

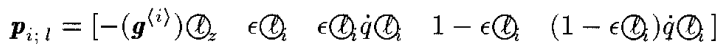

and $\epsilon_{i}$ is either 1 (when $\dot{q}_{i}>0$ ) or 0 (when $\dot{q}_{i}<0$ ). The minimal parameters can then be estimated recursively from link $n$ to link 1 by the least squares method if $\boldsymbol{P}_{i}^{* T} \boldsymbol{P}_{i}^{*}$ or $\boldsymbol{P}_{i}^{T} \boldsymbol{P}_{i}, i=1, \cdots, n$, are all of full rank. The trajectory from which the data are measured so that this condition is satisfied is named persistently exciting trajectory.

Using (30) and (31), it can be shown (see [19]) that a set of test trajectories of only rotational joint $i$ rotating with constant velocity is persistently exciting for rotational joint $i$ if

1) the trajectories are conducted for some configurations where joints except joint $i$ are held stationary and joint $i$ is not parallel to the gravitational direction;

2) the displacements of $q_{j}$ for $j=i+1, \cdots, k-1$ and one of $j \geq k$ are different for different configurations (to vary $b_{i, j}$ and $\nu_{i}^{*}$ or $\nu_{i}$ );

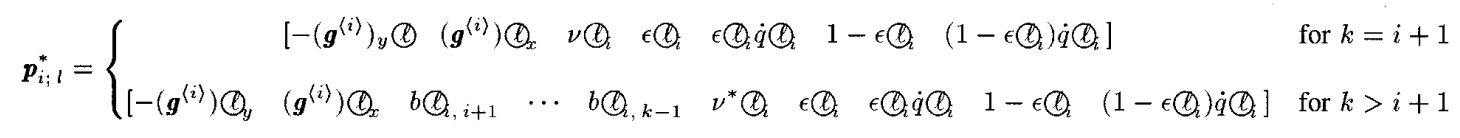


3) for each configuration, there are some trajectories with different angular velocities of joint $i$ covering the clockwise and the counterclockwise directions; and

4) the data are taken from some sampling points of each trajectory at which the vectors of $\left[\left(\boldsymbol{g}^{\langle i\rangle}\right) \bigotimes_{x},\left(\boldsymbol{g}^{\langle i\rangle}\right) \emptyset_{y}\right]^{T}$ are linearly independent.

For translational joint $i$, a set of trajectories of only joint $i$ moving with constant velocity is persistently exciting if there are at least five sampling points at which 1) joint $i$ is at two or more different configurations and not perpendicular to the gravitational direction (so that at least two of $\left(g^{\langle i\rangle}\right) \bigotimes_{z}$ are not equal), and 2) the joint velocities are different and cover the forward and the backward directions. Essentially, it is very easy to find a set of persistently exciting trajectories.

There is an extreme case for a translational joint. If the intersection angle of $\boldsymbol{u}_{i}$ and $\boldsymbol{g}$ for translational joint $i$ is invariant, the gravity load (i.e., $\left.\hat{m}_{i}^{*}\left(\boldsymbol{g}^{\langle i\rangle}\right)_{z}\right)$ is embodied in the friction and cannot be extracted since the first, second, and fourth columns of $\boldsymbol{P}_{i}$ [see (31)] are linearly dependent in this case. This case occurs when $i<r$ or when $r<i<s$ and $\boldsymbol{u}_{r} / / \boldsymbol{g}$. For the latter condition, $\delta_{r}$ is zero, so that $\hat{m}_{i}^{*}, i<s$, are unnecessary since $\boldsymbol{l}_{r}^{*}$ and $\boldsymbol{k}_{r}^{*}$ are not required for computing $i_{r}$. Note that if $\boldsymbol{u}_{i} \perp \boldsymbol{g}$, then $\sigma_{i}=0$, so that there is no embodying problem of the gravity load in the friction.

To make (29) still valid for the embodying case, it is just required to modify $\boldsymbol{w}_{i}$ in (29) to be $\boldsymbol{w}_{i}=\left[\hat{\sigma}_{i} \sigma_{i} \hat{m}_{i}^{*}, \hat{\lambda}_{1 i}^{+}, \lambda_{2 i}^{+}, \hat{\lambda}_{1 i}^{-}, \lambda_{2 i}^{-}\right]^{T}$ where $\hat{\sigma}_{i}=0$ for $i<r$ or for $r<i<s$ and $\boldsymbol{u}_{r} / / \boldsymbol{g}$, otherwise $\hat{\sigma}_{i}=1$ and

$$
\begin{aligned}
& \hat{\lambda}_{1 i}^{+}=\lambda_{1 i}^{+}+\left(1-\hat{\sigma}_{i}\right) K_{i} \sigma_{i} \hat{m}_{i}^{*}\left(\boldsymbol{g}^{\langle i\rangle}\right)_{z} \\
& \hat{\lambda}_{1 i}^{-}=\lambda_{1 i}^{-}+\left(1-\hat{\sigma}_{i}\right) K_{i} \sigma_{i} \hat{m}_{i}^{*}\left(\boldsymbol{g}^{\langle i\rangle}\right)_{z} .
\end{aligned}
$$

Corollary 4: For the same manipulator and the same notation in Theorem 3, if only one joint of the manipulator moves with a constant joint velocity at a time, a set of the minimal parameters for determining the armature currents $i$ for $\delta_{r} \neq 0$ is the union of $\left\{\delta_{i} K_{i}^{*}\left(\boldsymbol{k}_{i}^{*}\right)_{x}, \delta_{i} K_{i}^{*}\left(\boldsymbol{k}_{i}^{*}\right)_{y}, i=1, \cdots, n, \sigma_{j} K_{j} \hat{m}_{j}^{*}, \eta_{j} / \eta_{j+1}, j=\right.$ $\left.r, \cdots, n-1, \sigma_{n} K_{n} \hat{m}_{n}^{*}\right\}-\{0\}$ and $\left\{\lambda_{1 j}^{+}, \lambda_{2 j}^{+}, \lambda_{1 j}^{-}, \lambda_{2 j}^{-}, j=\right.$ $r, \cdots, n\}+\left\{\hat{\lambda}_{1 i}^{+}, \lambda_{2 i}^{+}, \hat{\lambda}_{1 i}^{-}, \lambda_{2 i}^{-}, i<r\right\}$.

If $\delta_{r}=0$, then $\eta_{r} / \eta_{r+1}, \cdots, \eta_{s-1} / \eta_{s}$ are redundant parameters, and $\sigma_{i} \hat{m}_{i}^{*}, \lambda_{1 i}^{+}$and $\lambda_{1 i}^{-}$for translational joint $i, r<i<s$, are replaced with $\hat{\lambda}_{1 i}^{+}$and $\hat{\lambda}_{1 i}^{-}$.

Algorithm: Identify the minimal parameters from joint $n$ to joint 1 .

Step 1) Let $i=n$

Step 2) If joint $i$ is a rotational joint, hold the manipulator at some configurations where joint $i$ is not parallel to the gravitational direction and $q_{j}$ for all $i<j<k$ and one of $j \geq k$ are different for different configurations, where $k$ is the next rotational joint behind joint $i$. For each configuration, rotate joint $i$ with some constant joint velocities in both clockwise and counterclockwise directions to take the data of the armature current $i_{i}$ with respect to the joint displacements and velocities.

If joint $i$ is a translational joint, hold joint $i$ at two or more configurations (if possible) and not perpendicular to the gravitational direction, then move joint $i$ alone with some constant velocities in both forward and backward directions to take the data of the armature current $i_{i}$ with respect to the joint displacements and velocities.

Step 3) If $i<n$, go to Step 4). Compute $g^{\langle n\rangle}$ for each sampling point. Use (29) to estimate $\left(\boldsymbol{k}_{n}^{*}\right)_{x},\left(\boldsymbol{k}_{n}^{*}\right)_{y}$ and the friction parameters (if joint $n$ is a rotational joint) or $\hat{m}_{n}^{*}$ and the friction parameters (if joint $n$ is a translational joint) by a standard least squares method.

Set $i=n-1$, go to Step 2).
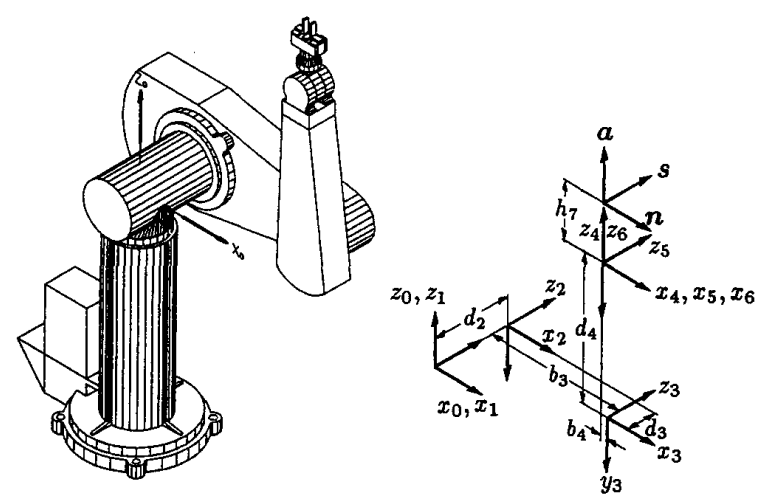

\begin{tabular}{lcccccc}
\hline \hline $\begin{array}{l}\text { Link } \\
\text { (Type) }\end{array}$ & Frame & $\theta$ & $\beta$ & $\begin{array}{c}b \\
(\mathrm{~m})\end{array}$ & $\begin{array}{c}d \\
(\mathrm{~m})\end{array}$ \\
\hline 1 & $(\mathrm{R})$ & $E_{0}$ & $q_{1}$ & $0^{\circ}$ & 0. & 0. \\
2 & $(\mathrm{R})$ & $E_{1}$ & $q_{2}$ & $-90^{\circ}$ & 0. & 0.2435 \\
3 & $(\mathrm{R})$ & $E_{2}$ & $q_{3}$ & $0^{\circ}$ & 0.4318 & -0.0934 \\
4 & $(\mathrm{R})$ & $E_{3}$ & $q_{4}$ & $90^{\circ}$ & -0.0203 & 0.4331 \\
5 & $(\mathrm{R})$ & $E_{4}$ & $q_{5}$ & $-90^{\circ}$ & 0. & 0. \\
6 & $(\mathrm{R})$ & $E_{5}$ & $q_{6}$ & $90^{\circ}$ & 0. & 0. \\
& $E_{6}$ & $0^{\circ}$ & $0^{\circ}$ & 0. & $h_{7}$ \\
\hline & $E_{E}$ & & &
\end{tabular}

Fig. 3. PUMA 560 arm and its normal driving-axis coordinate system.

Step 4) Compute $\boldsymbol{g}^{\langle i\rangle}$ for each sampling points. If joints $i$ and $i+1$ are rotational joints, compute $l_{i}^{*}$ recursively by using (24) and then $\nu_{i}$ by (26); then use (29) to estimate $\left(\boldsymbol{k}_{i}^{*}\right)_{x},\left(\boldsymbol{k}_{i}^{*}\right)_{y}, \eta_{i} / \eta_{i+1}$ and the friction parameters by a standard least squares method. If joint $i$ is a rotational joint and joint $k, k>i+1$, is the next rotational joint behind joint $i$, compute $b_{i, i+1}, \cdots, b_{i, k-1}$ and $\nu_{i}^{*}$ by (27) and (28) and then use (29) to estimate $\left(\boldsymbol{k}_{i}^{*}\right)_{x},\left(\boldsymbol{k}_{i}^{*}\right)_{y}, \eta_{i} / \eta_{i+1}, \cdots, \eta_{i} / \eta_{k}$ and the friction parameters by a standard least squares method. Finally, calculate out $\eta_{i+1} / \eta_{i+2}, \cdots, \eta_{k-1} / \eta_{k}$.

If joint $i$ is a translational joint, directly use (29) to estimate $\hat{m}_{i}^{*}$ and the friction parameters.

If $i=1$, stop. Otherwise set $i=i-1$ and go to Step 2).

It should be remarked that if some parameters in (29) are zero, the corresponding columns in $\boldsymbol{P}_{i}^{*}$ or $\boldsymbol{P}_{i}$ are removed in the identification procedure. The identification algorithm has been verified by a computer simulation on the Stanford arm, in which the measurement errors are ignored. To see the influence of the measurement errors, we present an experiment in the following section.

\section{EXPERIMENT}

The experiment is conducted on a PUMA 560 arm whose normal coordinate system is shown in Fig. 3. The six joints are all rotational and the first one is parallel to the gravitational direction, i.e., $r=$ $1, s=2$ and $\delta_{1}=0$. According to Corollary 4 , the minimal parameters are $\left(\boldsymbol{k}_{6}^{*}\right)_{x},\left(\boldsymbol{k}_{6}^{*}\right)_{y},\left(\boldsymbol{k}_{i}^{*}\right)_{x},\left(\boldsymbol{k}_{i}^{*}\right)_{y}, \eta_{i} / \eta_{i+1}, i=2, \cdots, 5$ and $\lambda_{1 j}^{+}, \lambda_{2, j}^{+} \lambda_{1 j}^{-}, \lambda_{2 j}^{-}, j=1, \cdots, 6$.

The identification algorithm requires two configurations for joints $2,3,4$, and 5 , and one configuration for joints 1 and 6 since there are no translational joints. At the home configuration, $q=\left[0^{\circ},-90^{\circ}, 90^{\circ}, 0^{\circ}, 0^{\circ}, 0^{\circ}\right]^{T}$, only joint 4 and joint 6 are parallel to the gravitational direction. We then select the first configurations for the tests of joints 4,5 , and 6 all to be 


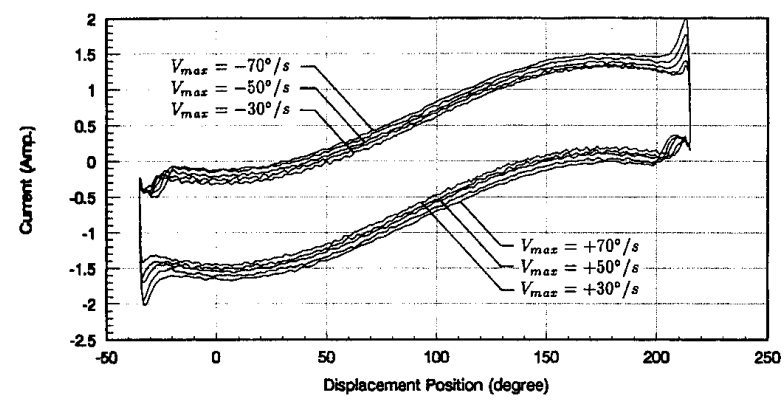

(a)

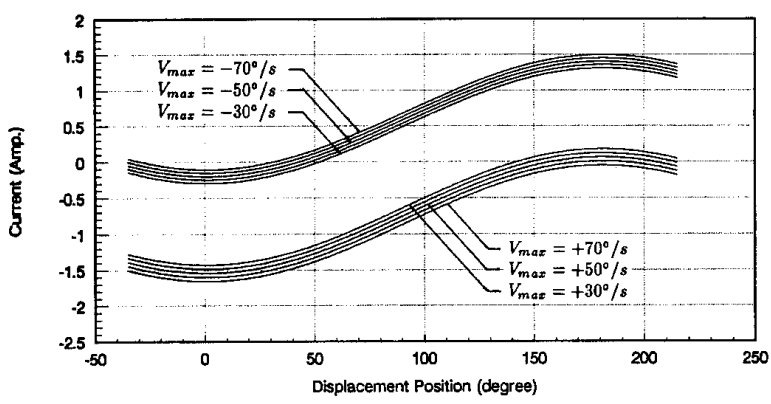

(b)

Fig. 4. Armature currents with respect to the joint positions for joint 3: (a) measured values, and (b) computed values with the identified parameters.

$q=\left[0^{\circ},-90^{\circ}, 0^{\circ}, 0^{\circ}, 0^{\circ}, 0^{\circ}\right]^{T}$. The second one for joint 5 is only to change $q_{6}$ to $90^{\circ}$ and that for joint 4 is to change $q_{5}$ to $90^{\circ}$. The first configurations for joints 1,2 , and 3 are the home configuration, while the second ones for joints 2 and 3 are, respectively, $q=\left[0^{\circ},-90^{\circ}, 150^{\circ}, 0^{\circ}, 0^{\circ}, 0^{\circ}\right]^{T}$ and $\boldsymbol{q}=\left[0^{\circ},-90^{\circ}, 90^{\circ}, 0^{\circ}, 90^{\circ}, 0^{\circ}\right]^{T}$. This makes a set of persistently exciting trajectories.

To achieve a stiff controller for the identification process (holding a constant speed over a large motion range), we implement the following discrete-time control law on each joint [5]

$$
\begin{gathered}
\ddot{q}_{i}^{*}(t)=K_{D}\left(-\dot{q}_{i}(t)+\frac{K_{P}}{K_{D}} V\left(q_{d i}(t)-q_{i}(t)\right)\right) \\
i_{i}(t)=J_{i} \ddot{q}_{i}^{*}(t)+i_{i}(t-\Delta t)-J_{i}\left(\frac{\dot{q}_{i}(t)-\dot{q}_{i}(t-\Delta t)}{\Delta t}\right)
\end{gathered}
$$

where

$$
V=\min \left(1, \frac{V_{\max }}{\frac{K_{P}}{K_{D}}\left\|q_{d i}(t)-q_{i}(t)\right\|}\right)
$$

$\dot{q}_{i}(t)=\left\{q_{i}(t)-q_{i}(t-\Delta t)\right\} / \Delta t, q_{d i}$ is the desired displacement of joint $i, \Delta t$ is the sampling time, $K_{D}$ and $K_{P}$ are control gains and are usually set to $K_{D}=2 \sqrt{K_{P}}$, and $J_{i}$ is a gain adjustable to balance the motor inertia. The controller (35) is called load compensator with acceleration feedback [29] and is equivalent to the robust independent joint controller of Hsia et al. [11]. The analyses in [11] and [29] showed that this control law is very suitable for the present experiment. On the other hand, (34) is a PD controller with a velocity constraint [12]. Due to (34), the joint motion can be kept at a constant velocity (i.e., $V_{m a x}$ ) in a wide range for a large step input.

For each of the above mentioned configurations, we let the corresponding joint rotate in a wide range of $250^{\circ}$ (from $-125^{\circ}$ to $+125^{\circ}$ and from $+125^{\circ}$ to $-125^{\circ}$ ) by inputting a step command in the

\begin{tabular}{|c|c|c|c|c|c|c|}
\hline & Link 1 & Link 2 & Link 3 & Link 4 & Link 5 & Link 6 \\
\hline$\left(k_{i}^{*}\right)_{x} \times 9.8(\mathrm{amp})$ & - & 2.191535 & 0.02212 & -0.03060 & -0.01424 & -0.00053 \\
\hline$\left(\boldsymbol{k}_{i}^{*}\right)_{y} \times 9.8(\mathrm{amp})$ & - & 0.00371 & 0.80144 & 0.01287 & 0.13828 & 0.00043 \\
\hline$\eta_{i} / \eta_{i+1}$ & - & -0.57450 & 0.10676 & 0.14463 & 0.000002 & - \\
\hline$\lambda_{1 i}^{+}$(amp) & 0.71678 & 0.54814 & -0.45359 & -0.16843 & -0.18605 & -0.16082 \\
\hline$\lambda_{2 i}^{+}(\mathrm{amp}-\mathrm{s} / \mathrm{m})$ & 0.00222 & 0.00677 & -0.00573 & -0.00133 & -0.00290 & -0.00181 \\
\hline$\lambda_{1 i}^{-}(\mathrm{amp})$ & -0.28355 & -0.98279 & 0.36250 & 0.22914 & 0.27428 & 0.23237 \\
\hline$\lambda_{2 i}^{*}(\mathrm{amp}-\mathrm{s} / \mathrm{m})$ & 0.00332 & 0.00291 & -0.00475 & -0.00121 & -0.00195 & -0.00238 \\
\hline $\begin{array}{l}\text { Standard (amp) } \\
\text { Deviation (amp }\end{array}$ & 0.0225 & 0.0293 & 0.0149 & 0.0187 & 0.0167 & 0.00636 \\
\hline
\end{tabular}

TABLE I

Identified VALues of the Minimal Parameters of the PUMA 560 ARM

control law (34)-(35) for the joint (the other joints are held stationary) while setting $V_{\max }$ to some values from $\pm 30 \%$ to $\pm 70 \%$. The armature current history is recorded during the motion. In any case, the current fluctuates. The gain $J_{i}$ and the sampling time $\Delta t$ are adjusted by a trial and error method to make the amplitude of the current fluctuation small while the joint velocity is still kept at the maximal velocity. Some current and velocity histories with respect to the displacements for joint 3 are shown in Fig. 4(a). The experiment results also demonstrate that the nonsymmetric friction model is quite well for the robot.

The measured data are given to the identification algorithm to get the values of the minimal parameters, which are listed in Table I. The HFTI algorithm (a QR-method) in [14] is used to obtain the least squares solutions for the present identification procedure. The minus signs of the friction parameters for links 3-6 are due to the facts that the directions of our joint coordinates for joints 3-6 are opposite to those of the corresponding motors set by the manufacturer. The value of $\eta_{5} / \eta_{6}$ in Table I seems incredibly small. The values of $\boldsymbol{k}_{6}^{*}$ are so small that $\nu_{5}$ in matrix $\boldsymbol{P}_{5}^{*}$ [see (30)] is negligible in comparison with other terms. This results in that the linear equations (29) are nearly rank-deficient. In fact, $\eta_{5} / \eta_{6}$ can be any finite value (less than $10^{4}$ ) without significantly affecting the residuals. In comparison with the values of $\boldsymbol{k}_{5}^{*}$, the $\mathrm{x}$ - and $\mathrm{y}$-components of $\boldsymbol{k}_{6}^{*}$ can be seen as redundant in the least squares sense, as can $\eta_{5} / \eta_{6}$. The numerical technique in the HFTI algorithm will set nearly rank-deficient matrix to be rank-deficient by a tolerance setting. For a rank-deficient matrix, the HFTI algorithm finds the minimum length solution (i.e., pseudoinverse solution) of the least squares problem [14]. Thus, the resulting value of $\eta_{5} / \eta_{6}$ is zero (or close to zero due to roundoff errors after transformation to the original coordinates). When $\nu_{5}$ is small, but not less than the tolerance, the result may be very large if the measurement errors are large (because the equation is inherently illconditioned). In the present experiment, the measurement errors are kept very small, so that the resulting value of $\eta_{5} / \eta_{6}$ is nearly zero (i.e., the equation is nearly consistent). That means the present method is robust to the case that the inertia constants of the outermost links are negligible small when the measurement errors are kept small such as was done in the present experiment.

The verification for the identified values of the inertia constants is not easy since the true values are not available. In the literature [2] and [32], the identified values were always used to compute the required actuator torques for some prescribed trajectories, and the results were compared with the measured torques to ensure that the identified values are acceptably accurate. When we let only one joint rotate with a constant joint speed, we also find that the measured armature currents match the computed ones with the identified values very well [e.g., see Fig. 4(b)].

As was mentioned above, the identified inertia constants are to be used in the feedback control law. To verify this validity, we implement the following PD feedback control with the gravity and 


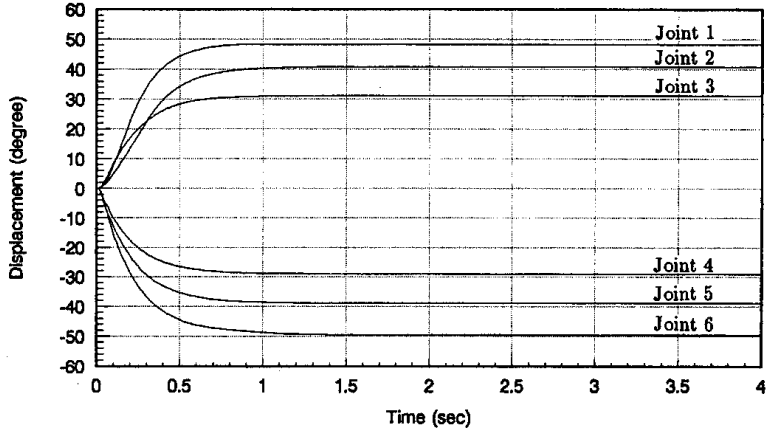

(a)

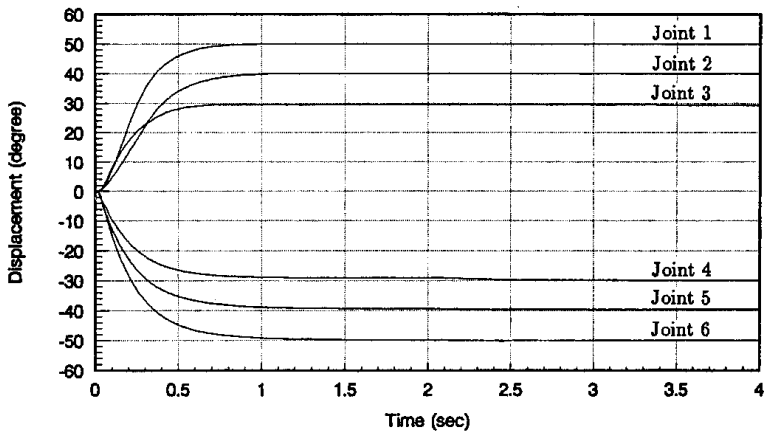

(b)

Fig. 5. Step responses of the PD feedback control for the initial position at the home configuration: (a) without compensation, and (b) with the gravity and friction compensation.

friction compensation for a step command

$$
\tau_{i}=-K_{D} \dot{q}_{i}+K_{P}\left(q_{d i}-q_{i}\right)+i_{i}
$$

where $\tau_{i}$ is the input command to the servo amplifier of motor $i$, and $i_{i}$ is computed by using (25) with the identified values of the minimal parameters. It should be remarked that the last three actuators of the PUMA 560 are coupled as [5]

$$
\begin{gathered}
\dot{q}_{S 4}=\frac{1}{r_{4}} \dot{q}_{4} \\
\dot{q}_{S 5}=\frac{1}{r_{5}} \dot{q}_{5}+r_{54} \dot{q}_{S 4} \\
\dot{q}_{S 6}=\frac{1}{r_{6}} \dot{q}_{5}+r_{64} \dot{q}_{S 4}+r_{65} \dot{q}_{S 5}
\end{gathered}
$$

where $\dot{q}_{S i}$ is the shaft velocity of the actuator motor $i$ and $r_{54}$, $r_{64}$, and $r_{65}$ are coupling gear ratios. To compute the friction compensation, $\dot{q}_{i}$ in (20) should be replaced with $r_{i} \dot{q}_{S i}$ for joints 5 and 6 since the friction arises from the motion of the gear train. The step responses of the control scheme (37) with and without the compensation (i.e., $i_{i}$ ) are shown in Fig. 5. The gains are $K_{D}=20$ and $K_{P}=100$, and the target position is $\boldsymbol{q}=$ $\left[50^{\circ}, 40^{\circ}, 30^{\circ},-30^{\circ},-40^{\circ},-50^{\circ}\right]^{T}$. The compensation computed by the identified parameters improves the steady-state errors. Because our model does not consider the stiction and low-velocity friction, there are some small steady-state errors in the step responses for the control with the compensation. The settling times for different joints are remarkably different. This can be explained by the fact that the coupling dynamics vary during the motion and only the gravity and friction (i.e., not all dynamics) are compensated for.

\section{CONCLUSION}

This note presents a parameter identification method for a class of nonlinear deterministic system such as a manipulator with unknown torque constants. The salient features of the application to a manipulator are to take into account the coupling of the torque constants and to identify the friction parameters at the same time. The experiment on the PUMA 560 arm presented in this note has a twofold purpose: illustration of the method and alleviation of the complicated error analysis. It is known that many factors such as measurement errors, quantization, and the selection of the persistently exciting trajectories will affect the results of the least squares method. The success of the present experiment lies in that the measurement errors are kept very small and the test trajectories cover a wide subspace of the workspace. In the engineering point of view, this experience is valuable.

It should be remarked that the main idea described in Section II can also be applied to the minimal parameters of the full dynamics of a manipulator. Some further effort is needed, however, to reform and simplify the full dynamics. It is also believed that there are other systems belonging to the class of nonlinear deterministic system (1), e.g., the following discrete multivariable system

$$
\begin{gathered}
a_{1} x(k)+a_{2} x(k-1)+a_{3} y(k)+a_{4} y(k-1)=b_{1} u_{1}(k) \\
a_{3} x(k)+a_{4} x(k-1)=b_{2} u_{2}(k)
\end{gathered}
$$

where $x$ and $y$ are state variables, $u_{1}$ and $u_{2}$ are input variables. The theory is then not restricted to manipulator systems.

\section{ACKNOWLEDGMENT}

The author would like to thank S.-L. Wu for his assistance in the experiment.

\section{REFERENCES}

[1] B. Armstrong, "Friction: experimental determination, modeling and compensation," in Proc. 1988 IEEE Conf. Robotics and Automation, 1988, pp. 1422-1427.

[2] C. C. Atkeson, C. H. An, and J. M. Hollerbach, "Estimation of inertial parameters of manipulator loads and links," Int. J. Robotics Res., vol. 5, no. 3, pp. 101-119, 1986.

[3] S. Benhlima, A. Barrraco, and B. Cuny, "Identification of dynamic parameters of robots," in Proc. 23rd Int. Symp. Industrial Robots, 1992, pp. 597-602.

[4] C. Canudas, K. J. Åström, and K. Braun, "Adaptive friction compensation in dc-motor drives," IEEE J. Robotics and Automation, vol. 3, pp. 681-685, 1987.

[5] J. C. Chen, S. K. Lin, and S. L. Wu, "A flexible control system for PUMA 560," in Proc. 15th National Symp. Automat. Contr., Taiwan, 1991, pp. 645-653.

[6] J. J. Craig, Introduction to Robotics: Manipulation and Control. Reading, MA: Addison-Wesley, 1986

[7] M. Gautier, F. Bennis, and W. Khalil, "The use of generalized links to determine the minimum inertial parameters of robots," J. Robotic Systems, pp. 225-242, 1990.

[8] M. Gautier and W. Khalil, "Identification of the minimum inertial parameters of robots," in Proc, 1989 IEEE Conf. Robotics and Automation, pp. 1529-1534, 1989.

[9] _Direct calculation of minimum set of inertial parameters of serial robots," IEEE Trans. Robotics and Automation, pp. 368-373, 1990.

[10] I. J. Ha, M. S. Ko, and S. K. Kwon, "An efficient estimation algorithm for the model parameters of robotic manipulators," IEEE Trans. Robotics and Automation, vol. 5, pp. 386-394, 1989.

[11] T. C. Hsia, T. A. Lasky, and Z. Guo, "Robust independent joint controller design for industrial robot manipulators," IEEE Trans. Industrial Electronics, vol. 38, pp. 21-25, 1991.

[12] O. Khatib, "A unified approach for motion and force control of robot manipulators: The operational space formulation," IEEE J. Robotics and Automation, vol. 3, pp. 43-52, 1987.

[13] P. K. Khosla and T. Kanade, "Parameter identification of robot dynamics," in Proc. IEEE Conf. Dec. and Contr., 1985, pp. 1754-1760. 
[14] C. H. Lawson and R. J. Hanson, Solving Least Squares Problems. Englewood Cliffs, NJ: Prentice-Hall, 1974.

[15] M. B. Leahy and G. N. Saridis, "Compensation of industrial manipulator dynamics," Int. J. Robotics Res., vol. 8, no. 4, pp. 73-84, 1989.

[16] S. K. Lin, "Microprocessor implementation of the inverse dynamic system for industrial robot control," in Proc. 10th 1FAC World Congress Automat. Contr., vol. 4, 1987, pp. 332-339.

[17] _ Identification of the inertia parameters of an industrial robot, National Science Council, Taiwan, NSC Proj. Rep. NSC80-0404-E-009$31,1991$.

[18] , "An identification method for estimating the inertia parameters of a manipualtor," J. Robotic Systems, vol. 9, no. 4, pp. 505-528, 1992.

[19] _ _ "Estimating minimal parameters of a manipulator for gravity load and friction," in Proc. 12th IFAC World Congress Automat. Contr., vol. 3, 1993, pp. 305-310.

[20] H. Mayeda, K. Osuka, and Kangawa, "A new identification method for serial manipulator arms," in Proc. IFAC 9th World Congress, 1984, p. 2429-2434.

[21] H. Mayeda, K. Yoshida, and K. Osuka, "Base parameters of dynamics models for manipulators with rotational and translational joints," in Proc. 1989 IEEE Conf. Robotics and Automation, 1989, pp. 1523-1528.

[22] _ - "Base parameters of manipulator dynamics models," IEEE Trans. Robotics and Automation, vol. 6, pp. 312-321, 1990.

[23] H. Mayeda and K. Ohashi, "Base parameters of dynamic models for general open-loop kinematic chains," in Proc. 5th Int. Symp. Robotics Research, 1990, pp. 271-278.

[24] A. Mukerjee and D. H. Ballard, "Self-calibration in robot manipulations," in Proc. 1985 IEEE Conf. Robotics and Automation, 1985, pp. 1050-1057.

[25] H. B. Olsen and G. A. Bekey, "Identification of robot dynamics," in Prob. 1986 IEEE Conf. Robotics and Automation, 1986, pp. 1004-1010.

[26] K. Otani, T. Kakizaki, and K. Kogure, "Dynamic parameter identification of an industrial robot and its application to trajectory controls," in Proc. 1992 IEEE/RSJ Conf. Intelligent Robots and Systems, 1992, pp. 990-997.

[27] B. Raucent, J. C. Samin, and P. Y. Willems, "Identification of dynamic parameters of robot manipulator from external measurements," European J. Mech. Eng., vol. 34, no. 1, pp. 21-26, 1989.

[28] B. Raucent, G. Bastin, J. C. Samin, and P. Y. Willems, "Identification of the barycentric parameters of robot manipulator from external measurements," Automatica, vol. 28, no. 5, pp. 1011-1016, 1992.

[29] R. Tsai and S. K. Lin, "Design and implementation of the torque control for a dc servo motor," in Proc. 14th National Symp. Automat. Contr., Taiwan, 1990, pp. 565-572.

[30] J. T. Wen and D. S. Bayard, "New class of control laws for robotic manipulators, part 1: Non-adaptive case," Int. J. Contr., vol. 47, pp. 1361-1385, 1988.

[31] H. West, E. Papadopoulos, S. Dubowsky, and H. Cheah, "A method for estimating the mass properties of a manipulator by measuring the reaction moments at its base," in Proc. 1989 IEEE Conf. Robotics and Automation, 1989, pp. 1510-1516.

[32] K. Yoshida, N. Ikeda, and H. Mayeda, "Experimental study of the identification methods for an industrial robot manipulator," in Proc. 1992 IEEE/RSJ Conf. Intelligent Robots and Syst., 1992, pp. 263-270.

\section{A Hardware Implementable Receding Horizon Controller for Constrained Nonlinear Systems}

Shin-Yeu Lin

Abstract-We present a two-phase parallel computing method for obtaining an implementable receding horizon control solution for constrained nonlinear systems. The phase 1 method solves a feasibility problem to find an approximate open-loop admissible control and horizon pair. The phase 2 method successively improves the admissible control to obtain an implementable open-loop receding horizon control solution. We briefly sketch an approach to realizing this two-phase method using VISI array processors. Solution times for simulation examples estimated on the basis of current VLSI technology confirm that our controller is well suited to the stabilization of real-time processing, fast, constrained nonlinear systems.

\section{INTRODUCTION}

For a nonlinear system with control constraints described by $\dot{x}(t)=f^{0}(x(t), u(t)), u(t) \in \Omega$, where $f^{0}: \mathfrak{R}^{n} \times \mathfrak{R}^{p} \rightarrow \mathfrak{R}^{n}$ is twice continuously differentiable and satisfies $f^{0}(0,0)=0$ and $\Omega$ is the set of admissible controls, Mayne and Michalska showed in [1] that feedback stabilization can be achieved by a conceptual receding horizon control. Subsequently, in [2] they proposed an implementable receding horizon controller which employed a hybrid system, $\dot{x}(t)=$ $f^{0}(x(t), u(t))$, when $x(t) \notin W$; otherwise $\dot{x}(t)=A x(t)$, where $A=f_{x}^{0}(0,0)+f_{u}^{0}(0,0) K$ is formed by applying a linear feedback control $u=K x$ to the linearized system in a neighborhood $W$ with small enough radius and centered at origin. Their algorithm for this implementable receding horizon controller first calculates an admissible control and horizon pair

$$
\left[u_{0}, t_{f, 0}\right] \in Z_{W}\left(x_{0}\right)
$$

where the initial state $x_{0} \notin W$ is assumed, the set $Z_{W}(\cdot): Z_{W}(x) \equiv$ $\left\{u \in S, t_{f} \in(0, \infty) \mid x^{u}\left(t+t_{f} ; x, t\right) \in \delta W\right\}$, where $S$ denotes the set of all piecewise continuous functions, and $\delta W$ denotes the boundary of $W$. The algorithm then sets $h=0, t_{h}=0$ and performs the following process repeatedly to yield the receding horizon feedback control: It applies the obtained control $u_{h}$ for $x \notin W$ or the linear feedback control $K x$ for $x \in W$ to the real system over $\left[t_{h}, t_{h}+\Delta t\right]$, where $\Delta t \in(0, \infty)$. Let $x_{h+1}$ be the resulting state at $t_{h+1}\left(\equiv t_{h}+\Delta t\right)$, then if $x_{h+1} \in W$, the algorithm switches the control to $u=K x$ over $\left(t_{h+1}, \infty\right)$; otherwise, it calculates an improved control and horizon $\left[u_{h+1}, t_{f, h+1}\right]$ in the sense that

$$
\begin{array}{r}
{\left[u_{h+1}, t_{f, h+1}\right] \in Z_{W}\left(x_{h+1}\right), \text { and } V\left(x_{h+1}, t_{h+1}, u_{h+1}, t_{f, h+1}\right)} \\
\leq V\left(x_{h+1}, t_{h+1}, u_{h}, t_{f, h}-\Delta t\right)
\end{array}
$$

where $V\left(x, t, u, t_{f}\right) \equiv \int_{t}^{t+t_{f}}(1 / 2)\left(\left\|x^{u}(\tau ; x, t)\right\|_{Q}^{2}+\|u(\tau)\|_{R}^{2}\right)$ $d \tau+\int_{t+t_{f}}^{\infty}(1 / 2)\left(\left\|x_{L}\left(s ; x^{u}\left(t+t_{f} ; x, t\right)\right)\right\|_{Q}^{2}\right) d s$, in which $R$ and $Q$ are positive definite matrices, $\|y\|_{A}^{2}$ denotes $y^{T} A y$, and $x_{L}$ denotes the state trajectory in region $W$ with feedback control $u=K x$.

Mayne and Michalska showed in [2] and [3] that the above implementable receding horizon controller is globally asymptotically

Manuscript received October 10, 1992; revised September 24, 1993 and December 2, 1993. This work was supported by National Science Council of Taiwan under Grant NSC 82-0404-E009-356.

The author is with the Department of Control Engineering, National Chiao Tung University, Hsinchu, Taiwan.

IEEE Log Number 9402736. 